\title{
Wireless power transfer with on-chip inductor and class-E power amplifier for implant medical device applications
}

\begin{abstract}
The popular use of biomedical implants has been going on in numerous applications that include the use of pacemakers and emerging retina prostheses, together with brain-computer interfaces. Other popular uses include drug delivery and smart orthopaedic implants. The avoidance of batteries or piercing wirings has made the wireless powering of these implantable devices highly attractive. In this paper, a design of a class-E power amplifier which has inductive loading appropriate for implant application was made using 130nm Silterra CMOS process at $2.4 \mathrm{~V}$ supply. A presentation of high-Q on-chip inductors is made as a way of improving the efficiency of the wireless power transfer (WPT) system at $37.5 \mathrm{MHz}$ industrial, scientific and medical (ISM) band. Wireless power transfer efficiency of 59-89\% is obtained for distance variation up to $10 \mathrm{~mm}$ of the implant coil from the transmit power coil. DC voltage of more $3 \mathrm{~V}$ is obtained for distance up to $10 \mathrm{~mm}$ of the implant coil; and the on-chip implant inductor measures a smaller size of $10 \mathrm{~mm} \times 10 \mathrm{~mm}$ making the design more suitable for the application of medical implant.
\end{abstract}

Keyword: Class-E power amplifier; Efficiency; Implant medical devices; Inductive coupling; Wireless power transfer 